\title{
A Pilot Phase II Study of Erlotinib for the Treatment of Patients with Relapsed/ Refractory Acute Myeloid Leukemia
}

\author{
Iman Abou Dalle ${ }^{\mathrm{a}}$ Jorge E. Cortes ${ }^{\mathrm{a}}$ Pramod Pinnamaneni ${ }^{\mathrm{a}}$ Betty Lamothe ${ }^{\mathrm{b}}$
} Adolfo Diaz Duque $^{\mathrm{a}}$ Jasleen Randhawa ${ }^{\mathrm{a}}$ Naveen Pemmaraju $^{\mathrm{a}}$ Elias Jabbour ${ }^{\mathrm{a}}$ Alessandra Ferrajoli $^{a}$ William G. Wierda ${ }^{a}$ Zeev Estrov ${ }^{a}$ Marina Konopleva ${ }^{a}$ Farhad Ravandi $^{\mathrm{a}}$ Yesid Alvarado $^{\mathrm{a}}$ Gautam Borthakur ${ }^{\mathrm{a}}$ Varsha Gandhia, b $^{\mathrm{a}}$ Hagop M. Kantarjian ${ }^{a}$

a Department of Leukemia, The University of Texas MD Anderson Cancer Center, Houston, TX, USA; ${ }^{b}$ Department of Experimental Therapeutics, The University of Texas MD Anderson Cancer Center, Houston, TX, USA

\section{Keywords}

Erlotinib · Acute myeloid leukemia · Relapsed/refractory AML

\begin{abstract}
Erlotinib, an epidermal growth factor receptor (EGFR) inhibitor, may have off-target activity inducing acute myeloid leukemia (AML) differentiation, possibly through SYK inhibition. We investigated erlotinib in a pilot phase II study for efficacy in relapsed/refractory AML patients at a dose of $150 \mathrm{mg}$ once daily in 28-day cycles. Twenty-nine patients were treated for a median of 29 days (range 12-142 days). Seven patients (24\%) received $>1$ cycle of therapy and 12 (41\%) discontinued treatment before day 28 due to disease progression. One patient (3\%) achieved complete remission and 2 (7\%) a $>50 \%$ reduction in blasts. The most common toxicities associated with erlotinib were fatigue in 10 patients (34\%), diarrhea in 10 (34\%), nausea in 8 (28\%), and rash in 7 (24\%). Only 2 patients (7\%) had study drug-related adverse events requiring dose reductions and eventual discontinuation. The main reason for treatment discontinuation was disease pro-
\end{abstract}

gression in 26 patients (90\%). All patients had died by the time of the last follow-up. Progression of disease was the primary cause of death in all patients. Median overall survival was 14 weeks (range 2.3-96.9 weeks) and median event-free survival was 5 weeks (range 1.7-21.0 weeks). Erlotinib as a single agent has limited clinical efficacy in patients with relapsed/refractory AML.

(c) 2018 S. Karger AG, Basel

\section{Introduction}

The treatment of relapsed/refractory acute myeloid leukemia (AML) remains an ongoing challenge with a very poor outcome, with only $10 \%$ of patients surviving for 3 years. Currently, the standard treatment includes intensive salvage chemotherapy in physically fit patients, leading to complete remission and subsequent allogeneic hematopoietic stem cell transplantation (HSCT) [1].

Trial registration: clinicaltrials.gov ID: NCT01664897.

Jorge Cortes, $\mathrm{MD}$

Department of Leukemia, The University of Texas MD Anderson Cancer Center PO Box 301402, Unit 428

Houston, TX 77230 (USA)

E-Mail jcortes@mdanderson.org 
However, this approach is not always feasible in many situations such as older age, comorbid conditions, poor performance status, early relapse, and adverse genetic factors. There is a need to identify and evaluate new therapeutic agents in clinical trials, whenever possible [2]. Over the past decade, a better understanding of the genetic landscape of AML has led to the discovery of important molecular abnormalities and biologic pathways that could serve as potential targets for AML treatment [3].

Erlotinib is a potent, selective, and reversible tyrosine kinase inhibitor that acts on epidermal growth factor receptor (HER1/EGFR). Based on the EURTAC trial, a phase 3 randomized study, in 2013, the FDA approved erlotinib (150 mg orally once daily) for the treatment of patients with untreated locally advanced or metastatic non-small-cell lung cancer (NSCLC) harboring activating EGFR mutations [4]. Interestingly, 2 case reports on patients receiving erlotinib for NSCLC with a simultaneous diagnosis of AML documented concomitant efficacy in NSCLC and AML $[5,6]$. In addition, gefitinib, another EGFR inhibitor, has been reported to induce differentiation in 3 AML cell lines, suggesting a possible off-target effect on myeloid leukemia cells $[7,8]$. Boehrer et al. [9, 10] later tested erlotinib's effects in AML and myelodysplastic syndrome (MDS), and found erlotinib to be more potent at inducing differentiation than other FDA-approved EGFR tyrosine kinase inhibitors. Furthermore, using a novel, integrated orthogonal proteomic and genetic approach to identify the off-target activity of erlotinib in AML, as EGFR is not widely expressed in AML, spleen tyrosine kinase (SYK) inhibition was identified as the potential off-target anti-AML mechanism [11]. SYK is a critical tyrosine kinase signaling molecule of activating Fc receptors [12] as well as B cell receptors (BCR) [13, 14]. SYK is highly expressed and phosphorylated in AML, and SYK inhibition has been reported to trigger the differentiation of AML cells [15]. To date, no SYK inhibitors have been clinically tested in AML. Based on these observations and reports, we hypothesized that erlotinib could have clinical activity in patients with AML, possibly through SYK inhibition, and we conducted a phase II clinical trial for patients with relapsed or refractory AML.

\section{Methods}

\section{Patient Eligibility}

Patients with AML, who were either refractory to/relapsed after previous therapy, were eligible for this study. Eligibility was also extended to those with MDS or chronic myelomonocytic leukemia (CMML) who failed treatment with hypomethylating agents and whose disease had progressed to AML. Additional inclusion criteria included an Eastern Cooperative Oncology Group performance status (ECOG-PS) of 0-2, adequate hepatic function (total bilirubin $\leq 2 \times \mathrm{ULN}$, ALT $\leq 2.5 \times \mathrm{ULN}$ ), and adequate renal function (creatinine $\leq 2 \times \mathrm{ULN}$ ). All patients were required to have a wash-out period of 2 weeks from previous chemotherapy, except in the case of rapidly progressing disease with no evidence of clinically significant toxic effects from the prior treatment. Patients with rapidly proliferative disease were allowed the use of hydroxyurea prior to study entry and for the first 4 weeks of study treatment. Both males and females with reproductive potential were required to practice effective contraception and all females with child-bearing potential had a negative pregnancy test prior to the initiation of the study. Patients with other known diseases (except carcinoma in situ) or concurrent severe/uncontrolled comorbidities (such as uncontrolled diabetes or hypertension, congestive heart failure NYHA Class III or IV, recent myocardial infarction within 6 months, chronic renal failure, active infection, or gastrointestinal disorders that could interfere with erlotinib absorption) were excluded. Those able to receive a stem cell transplant within 4 weeks were also excluded, as they would not be able to reach the 28-day mark. The study was approved by the Institutional Review Board of The University of Texas MD Anderson Cancer Center, and all patients signed an informed consent document in accordance with the Declaration of Helsinki. The study was registered in clinicaltrials.gov as No. NCT01664897.

\section{Treatment Regimen and Safety Monitoring}

Patients received erlotinib $150 \mathrm{mg}$ once daily in consecutive 28day cycles until clinically significant disease progression or unacceptable toxicity. Erlotinib was to be taken at approximately the same time each day with up to $200 \mathrm{~mL}$ of water $1 \mathrm{~h}$ before or $2 \mathrm{~h}$ after a meal. Erlotinib was not to be taken with grapefruit and any missed doses were not made up. Dose reductions to 100 and $50 \mathrm{mg}$ daily were indicated for patients with significant adverse events. Patients with persistent toxicity at the 50 -mg daily dose were removed from the study. No dose reductions or treatment interruptions were planned for hematologic parameters during the first 4 weeks of therapy. After 4 weeks, dose reductions for hematologic effects could be considered on an individual basis. Patients underwent treatment until there was progression of disease or drug toxicities that could not be controlled with dose adjustments.

\section{Evaluation}

Pretreatment evaluation involved obtaining a complete history, a physical examination, a complete blood count (CBC), blood chemistry, a pregnancy test if applicable, and bone marrow (BM) aspiration with cytogenetic and molecular testing, and was done within 14 days of initiation of the study. Evaluation during treatment consisted of physical exams and assessment of adverse events, and CBC and blood chemistry once weekly for the first 3 months and then every 2-4 weeks up to 6 months of therapy. BM aspiration was planned on day 28 ( \pm 7 days) and then every $2-3$ months for 1 year. Responders were patients who achieved complete remission (CR), CR with incomplete hematologic recovery (CRi), partial remission (PR), hematologic improvement (HI), or a morphologic leukemia-free state (MLF) according to the definitions of the International Working Group [16]. Adverse events were evaluated throughout the course of therapy according to the Common Terminology Criteria for Adverse Events (CTCAE) v4. 
Collection and Isolation of Peripheral Blood Mononuclear Cells

Peripheral blood was collected from consenting patients at baseline and during the course of erlotinib therapy. Only patients who agreed for optional laboratory investigations and signed informed consent forms participated in this study. Peripheral blood mononuclear cells (PBMCs) were separated by Ficoll-Hypaque density centrifugation according to the manufacturer's instructions (Atlanta Biologicals; Flowery Branch, GA, USA), and PBMC pellets were stored for further analysis.

\section{Cell Lines and Culture}

The AML cell lines, OCI-AML3, ML-1, KG1, and MOLM-13, were cultured in RPMI 1640 (Hyclone; Logan, UT, USA) containing $10 \%$ fetal bovine serum, and then tested for mycoplasma infection. The erlotinib inhibitory kinase effect was further assessed in KG1 and MOLM-13. Cell lines were left untreated or were treated with vehicle or an increasing dose of erlotinib (Selleckchem; Houston, TX, USA) for the indicated times and then harvested, and cell pellets were stored for further analysis.

\section{Western Blot Analysis}

Cell lysates were prepared as previously described [17], and then subjected to SDS-PAGE followed by immunoblotting with antibodies against SYK, phospho-SYK $\left(\mathrm{Tyr}^{352}\right)$, SRC, phosphoSRC family $\left(\mathrm{Tyr}^{416}\right)$, all from Cell Signaling Technology (Beverly, MA, USA), $\beta$-actin (Cytoskeleton; Denver, CO, USA) and phosphotyrosine from EMD Millipore (Corp. Billerica, MA, USA).

\section{Study Design}

This was a phase II, single-institution, single-arm, open-label study to assess the efficacy and safety of erlotinib in patients with refractory or relapsed AML. The starting dose was selected based on the standard dose for first-line therapy in patients with NSCLC. The historical experience in this patient population is a response rate of $<1 \%$ with standard therapy. Therefore, an overall response rate within the first 3 months of therapy of $20 \%$ was defined as a rate of interest for the further pursuit of this approach. A Simon's optimal 2-stage design was used: 14 patients enrolled in the 1 st stage; if $\geq 1$ responded, 15 more patients were to be enrolled in the 2nd stage, i.e., a total of 29 patients. These sample sizes are based on Simon's optimal 2-stage designs of $\alpha=0.05$ with a power of $95 \%$ ( $\beta=0.05)$ to test the null hypothesis that the overall response rate (ORR) will be $\leq 1 \%$ versus the alternative hypothesis that ORR will be $\geq 20 \%$. If at least 1 patient of the first 14 did not show a response, the study would have been terminated early for futility. If there were at least 2 responses achieved after the completion of the 2 nd stage, the study would conclude that erlotinib improves the response rate from 1 to $20 \%$. The probability of at least 2 responses under the null hypothesis $(\mathrm{ORR}=1 \%)$ is $3 \%$ while the probability of at least 2 responses under the alternative hypothesis (ORR $\geq 20 \%$ ) is $94.8 \%$.

\section{Results}

\section{Study Population}

From May 2013 to May 2014, 30 patients were enrolled in the study. One patient did not start therapy because of a rapid deterioration after obtaining informed consent, so
Table 1. Patient characteristics
Age, years

Male/female

Relapsed disease

Refractory disease

Secondary AML

Previous transplantation

White blood cells, $\times 10^{9} / \mathrm{L}$

Hemoglobin, g/dL

Absolute neutrophil count

Platelets, $\times 10^{9} / \mathrm{L}$

Bone marrow blasts, $\%$

Peripheral blasts, \%

Prior treatments

Cytogenetics

Complex

Diploid

Molecular profile

NPM1

IDH2

FLT3

IDH1

TP53

KIT

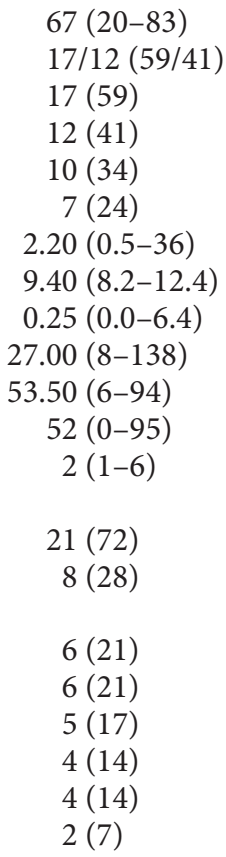

67 (20-83)

$17 / 12(59 / 41)$

17 (59)

$12(41)$

$10(34)$

$7(24)$

$2.20(0.5-36)$

$9.40(8.2-12.4)$

$0.25(0.0-6.4)$

$27.00(8-138)$

53.50 (6-94)

$52(0-95)$

2 (1-6)

$21(72)$

$8(28)$

$6(21)$

$6(21)$

5 (17)

4 (14)

4 (14)

2 (7)

Values are expressed as median (range) or $n(\%)$.

29 patients were evaluable for response and toxicity. The median age for the 29 patients was 67 years (range 20-83 years), and 17 (59\%) were males. Seventeen patients (59\%) had relapsed disease with a median duration of prior remission of 5 months (range 1-13 months) and 12 (41\%) had refractory disease. Ten patients (34\%) had secondary AML, 7 progressing from prior MDS and 3 after chemotherapy for other tumors. All patients had received previous treatments, with a median of 2 (range 1-6), and 7 (24\%) had previously received HSCT. Complex cytogenetics was identified in $72 \%$ of patients. Molecular abnormalities were identified in 17 of 28 patients (61\%) with evaluable molecular analysis, including NPM1 in $6(21 \%)$, IDH2 in 6 (21\%), FLT3 in 5 (17\%), IDH1 in 4 (14\%), TP53 in $4(14 \%)$, and KIT in $2(7 \%)$. Patient characteristics are summarized in Table 1.

\section{Outcomes}

Patients received therapy for a median of 29 days (range 12-142 days). Twelve patients (41\%) discontinued therapy before 28 days because of disease progression. Seven (24\%) received $>1$ cycle of therapy: 3 received 2 cycles, 2 received 3 cycles, and 1 each received 4 and 5
Abou Dalle et al. 


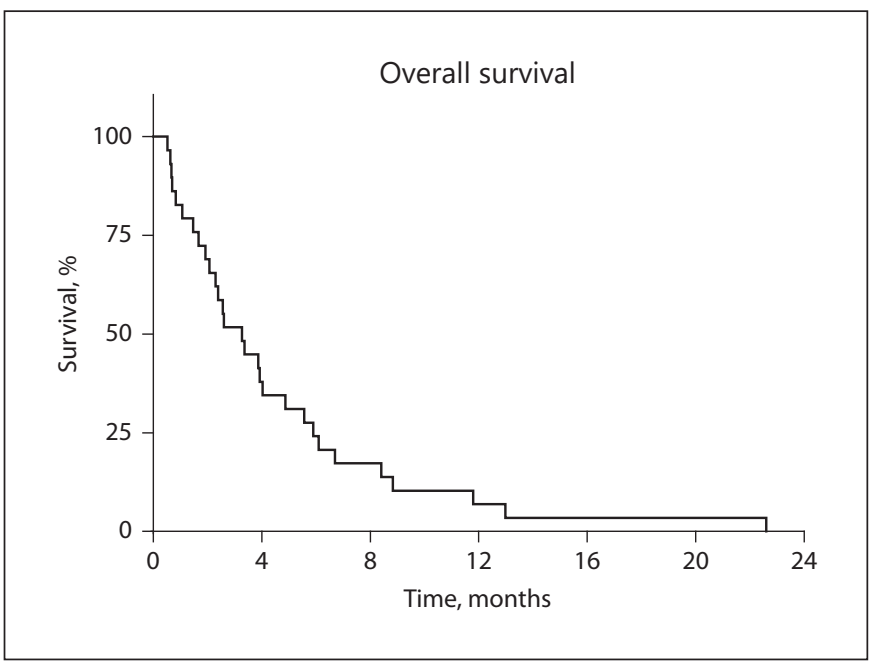

Fig. 1. Kaplan-Meier analysis of overall survival.

Table 2. Response to therapy

\begin{tabular}{lcc}
\hline Response & $n$ & $\%$ \\
\hline Complete remission & 1 & 3 \\
Partial remission & 0 & 0 \\
Hematologic improvement & 2 & 7 \\
Progression of disease & 26 & 90 \\
\hline
\end{tabular}

cycles, respectively. All 29 patients (100\%) eventually discontinued therapy: 26 (90\%) for disease progression, 2 (7\%) for adverse events (both after 2 cycles), and 1 (3\%) for noncompliance after 1 cycle.

One patient (3\%) achieved CR and 2 patients (7\%) had a $>50 \%$ reduction in BM blasts (from 91 to $23 \%$, and from 62 to $9 \%$, respectively) for an overall response rate of $10 \%$ (Table 2). The patient who achieved CR was a 60 -year-old male with complex cytogenetics who had previously been heavily treated with high-dose ara-C-containing regimens and received 2 allogeneic HSCTs. This patient received 5 cycles of therapy with erlotinib before progressing. The 2 patients with improvement in blasts experienced disease progression during the 2 nd cycle and were removed from the study. There was a temporary $\mathrm{HI}$ in a total of 9 patients $(31 \%)$, with an initial improvement in neutrophils in 8 patients from a median of 0.28 (range $0.07-0.78 \times 10^{9} / \mathrm{L}$ ) to 1.07 (range $0.62-2.22 \times 10^{9} / \mathrm{L}$ ), and in platelets in 2 patients (from 17 to $49 \times 10^{9} / \mathrm{L}$ and from 41 to $265 \times 10^{9} / \mathrm{L}$, respectively). Improvements were not

Erlotinib in the Treatment of Relapsed/ Refractory AML

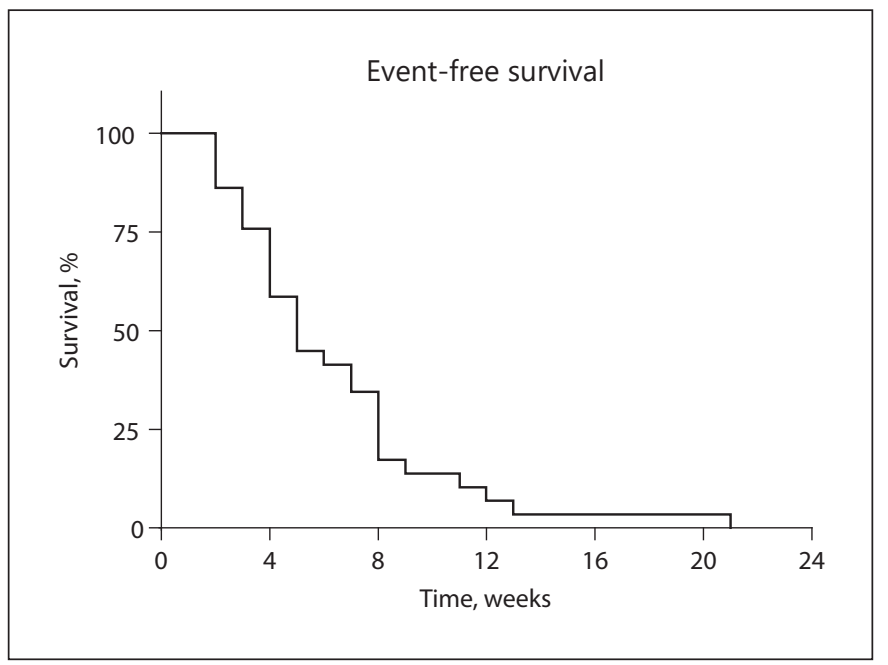

Fig. 2. Kaplan-Meier analysis of event-free survival.

significant, sustained, or associated with a blast response, so these patients are reported as having no response/progressive disease.

At the time of the last follow-up, all patients (100\%) had died, with a median survival of 3.5 months (range 0.6-24 months) from the start of their treatment. None of the patients died while on therapy. Progression of disease was the primary cause of death in all patients. The median overall survival (OS) was 14 weeks (range 2.3-96.9 weeks) (Fig. 1). The median event-free survival (EFS) was 5 weeks (range 1.7-21.0 weeks) (Fig. 2).

\section{Adverse Events}

The most common treatment-emergent adverse events were pneumonia in 14 patients (48\%), neutropenic fever in $13(45 \%)$, and shortness of breath in $11(38 \%)$, most of them considered as not related to the study drug. The most common adverse events considered possibly or probably related to erlotinib were fatigue in 10 patients (34\%), diarrhea in $10(34 \%)$, nausea in $8(28 \%)$, and rash in 7 (24\%). Overall, erlotinib was tolerated well and there were no treatment interruptions for toxicity. Only 2 patients (7\%) had study drug-related adverse events requiring dose reductions and eventual discontinuation: grade 3 fatigue in one and grade 3 diarrhea in the other, which did not improve with dose reductions to $50 \mathrm{mg}$ daily, eventually requiring discontinuation. One patient (3\%) developed a subdural hematoma not related to the study drug. All adverse events and toxicities are summarized in Table 3. 
Table 3. Adverse events and toxicities

\begin{tabular}{lclll}
\hline Adverse event & Total & Grade 1 & Grade 2 & Grade 3/4 \\
\hline Pneumonia & $14(48)$ & $0(0)$ & $0(0)$ & $14(48)$ \\
Neutropenic fever & $13(45)$ & $0(0)$ & $0(0)$ & $13(45)$ \\
Shortness of breath & $11(38)$ & $3(10)$ & $4(14)$ & $4(14)$ \\
Fatigue & $10(34)$ & $3(10)$ & $5(17)$ & $2(7)$ \\
Diarrhea & $10(34)$ & $4(14)$ & $5(17)$ & $1(3)$ \\
Nausea & $8(28)$ & $2(7)$ & $5(17)$ & $1(3)$ \\
Rash & $7(24)$ & $7(24)$ & $0(0)$ & $0(0)$ \\
Edema & $5(17)$ & $1(3)$ & $3(10)$ & $1(3)$ \\
Muscle aches & $5(17)$ & $0(0)$ & $4(14)$ & $1(3)$ \\
Watery eyes & $5(17)$ & $4(14)$ & $1(3)$ & $0(0)$ \\
Dizziness & $4(14)$ & $0(0)$ & $2(7)$ & $2(7)$ \\
Subdural hematoma & $1(3)$ & $0(0)$ & $1(3)$ & $0(0)$ \\
\hline
\end{tabular}

Values are expressed as $n(\%)$. munoreactive bands of lower molecular weight that increased concurrently by day 5 (Fig. 3c, asterisks). In addition, all 3 patient samples exhibited a similar pattern of total tyrosine phosphorylated proteins (Fig. 3d), with the exception that, in AML-064 and AML-122, a tyrosine phosphorylated protein (approx. $75 \mathrm{kDa}$ ) was detected at baseline and vanished after 5 days on erlotinib therapy (Fig. 3d, asterisks). Furthermore, as a quality control for the Western blot analysis, we concurrently analyzed the 3 patient samples with samples from the AML cell lines KG1 and MOLM-13, either untreated or treated with 5 and $10 \mu \mathrm{M}$ erlotinib for $6 \mathrm{~h}$ (Fig. 3c, d). Due to the limited number of patient samples available in this pilot study, no conclusion can be drawn to exclude or validate SYK and SRC as in vivo kinase candidates targeted by erlotinib in AML.

\section{Discussion}

There is a pressing need for more therapeutic options for patients with relapsed and refractory AML regardless of age. Not only are the outcomes for this patient population very poor, but standards of treatment have not changed in decades. Better understanding of the biology of AML has led to the development of many new targeted therapies being recently approved by the FDA, such as midostaurin for FLT3 mutant AML and enasidenib for IDH2 mutant AML [1]. These options, however, are only applicable to a minority of patients with AML. In the light of preclinical data showing that an EGFR kinase inhibitor can induce terminal differentiation of AML cells, we developed a pilot phase II study to evaluate the efficacy of single-agent erlotinib in patients with relapsed/refractory AML.

Unfortunately, erlotinib demonstrated limited clinical efficacy in this trial for patients with heavily pretreated refractory or relapsed AML. Three patients had some evidence of a response to therapy, including 1 that achieved a CR. This patient had minimal disease burden at baseline, and achieved a remission that lasted 5 months. Two other patients had a significant but transient decrease in BM blasts. Most other patients progressed rapidly, frequently before the end of the first cycle. It is possible that the burden of disease may limit the potential benefit of erlotinib as a single agent.

A study exploring erlotinib in patients with MDS/ AML after azacytidine failure demonstrated a $20 \%$ overall response with a 5-month median duration of response [21]. All 6 responders had a WHO classification of RAEBduring therapy (Fig. 3c). Notably, we observed 2-3 im- 


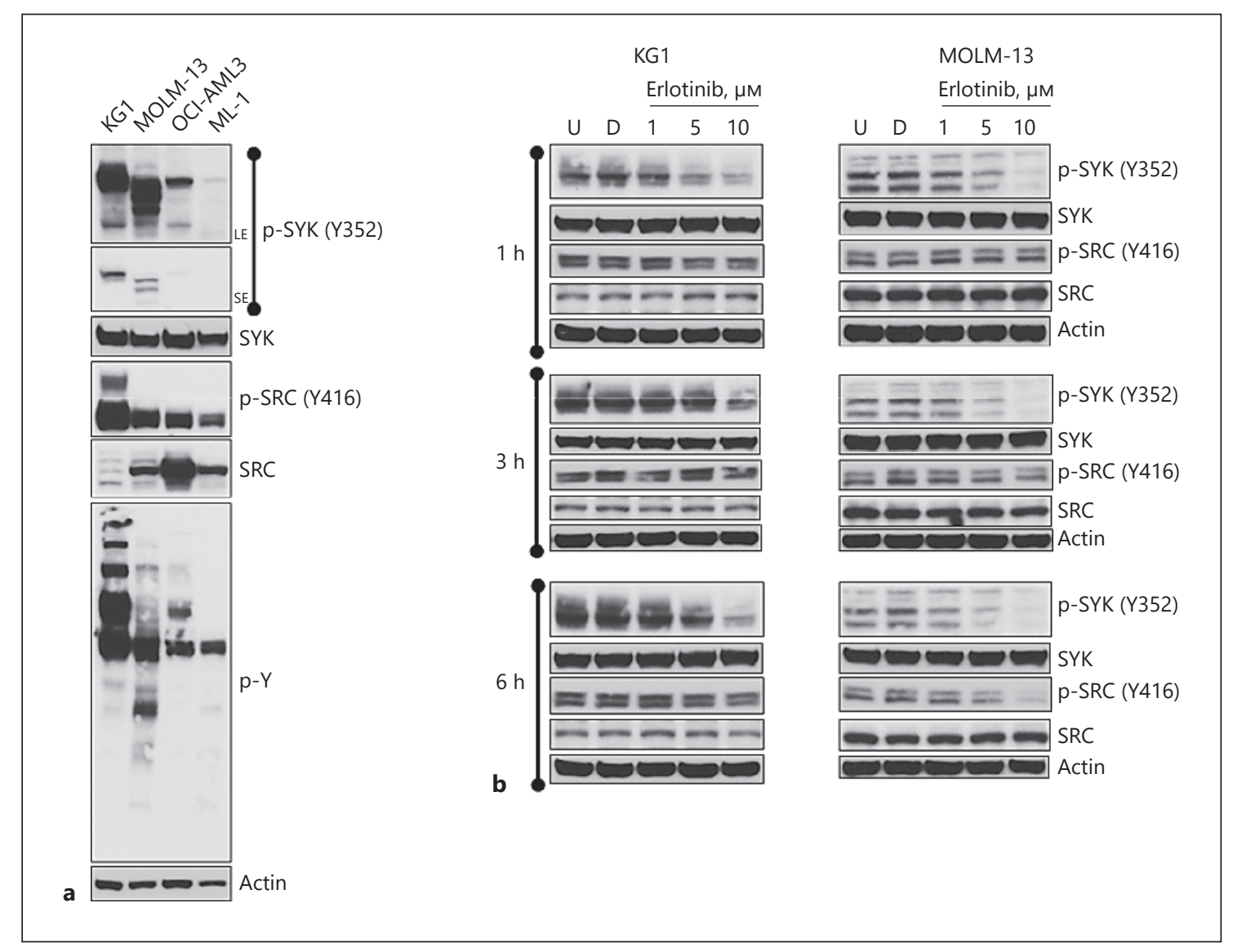

Fig. 3. Evaluation of phosphorylated SYK and SRC status in AML samples before and following erlotinib treatment. a SYK and SRC proteins are highly phosphorylated in KG1 and MOLM-13. Lysates from 4 AML cell lines were evaluated by Western blot with the indicated antibodies. b Erlotinib treatment resulted in attenuation of SYK and SRC phosphorylation in a dose- and time-dependent manner. KG1 and MOLM-13 cell lines were left untreated $(\mathrm{U})$, or were treated with vehicle (D; DMSO), or the indicated concentrations of erlotinib. At the indicated times, the cells were collected, lysed, and cell lysates were subjected to Western blot analy- sis with the indicated antibodies. c, d Assessment of SYK and SRC phosphorylation status in PBMCs isolated from 3 AML patients at baseline and during erlotinib therapy. PBMCs were harvested at baseline (pre), and at the indicated times during erlotinib treatment. KG1 and MOLM-13 were treated for $6 \mathrm{~h}$ as indicated in $\mathbf{b}$. c Concurrently, cell pellets from PBMCs and cell lines were lysed and subjected to Western blot analysis with the indicated antibodies. d Total tyrosine phosphorylated proteins ( $\mathrm{p}-\mathrm{Y}$ ) were also evaluated by Western blot analysis. U, untreated; D, DMSO; SE, short exposure; LE, long exposure.

(Figure continued on next pages.)
2. No response was seen in those who were classified as AML and all but 1 of the patients who responded had a normal karyotype. In another small pilot study conducted at the Indiana University School of Medicine, $11 \mathrm{AML}$ patients were treated with the same dose of erlotinib [22], but this study was also not able to demonstrate clinical efficacy. Whether this represents the inability of erlotinib to control a more proliferative disease and/or higher disease burden, or whether specific molecular events in MDS might make this disease more prone to responding to erlotinib, remains to be defined. Interestingly, Mahmud et al. [23] identified a subset of patients (15\%) with AML expressing EGFR at the protein level. This may indicate that erlotinib might be effective in this selected subset.

Based on preclinical data, SYK inhibition is the suspected primary off-target anti-AML mechanism of erlotinib that causes AML differentiation in vitro. Phosphorylated SYK can be used as a biomarker of response and is correlated with an unfavorable profile of AML regardless of age, cytogenetics, and white blood cells [24]. However, the preclinical data is not expansive and the exact biology is not completely known. We were able to assess SYK phosphorylation in only 3 patient samples enrolled in this trial. Two showed SYK phosphorylation at base- 


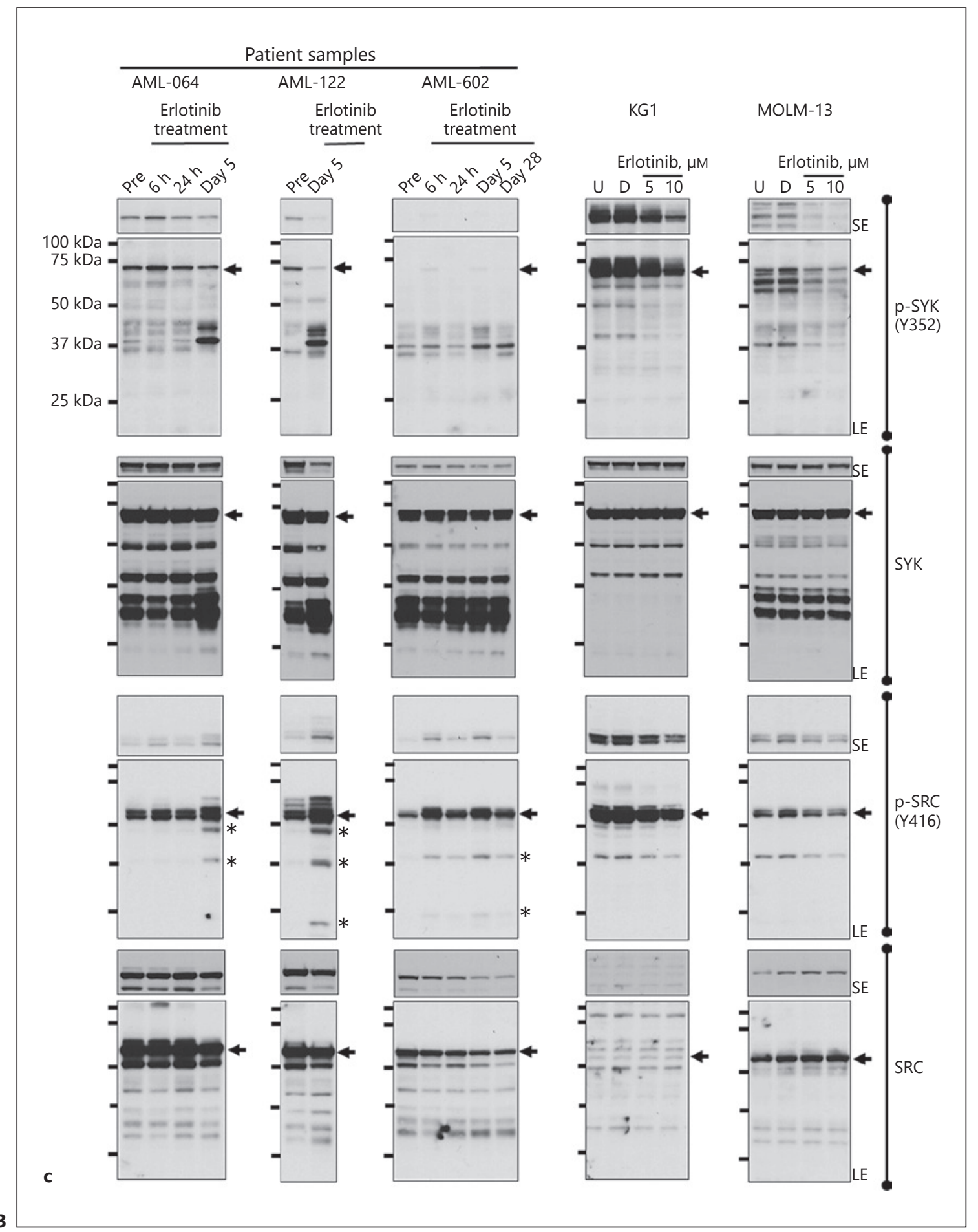




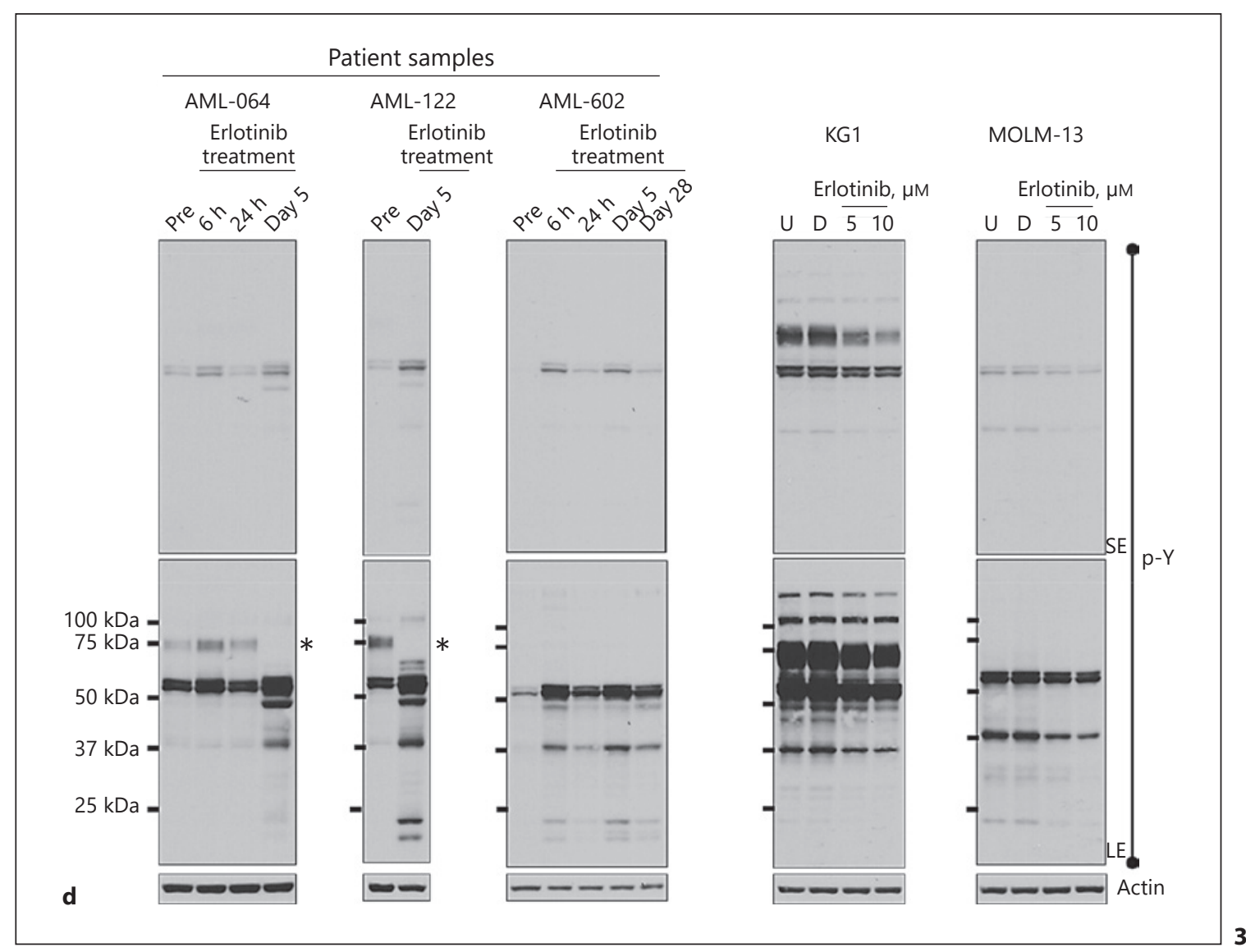

line, with no significant changes during the course of therapy for one of them, while the other demonstrated a decrease in phosphorylation and total protein, and we could not find any significant correlation with clinical outcome.

Even when phosphorylation is demonstrated, the dependency of the leukemic cells on SYK may vary from patient to patient, and an adequate response may require combination therapy as sustained differentiation is likely not along a linear pathway. Erlotinib in combination with azacytidine can be synergistic in AML cell lines by increasing intracellular concentration of azacytidine [25]. This combination is worth testing in vivo as a potential antileukemic treatment strategy. It is also possible that the given in vivo concentrations of erlotinib may not be enough to consistently provide SYK inhibition or multiple kinase inhibition. There may also be different levels of SYK activation in specific AML subsets, such as those with FLT3 mutations. For instance, a study by Puissant et al. [26] suggested increased sensitivity to SYK inhibition in samples with FLT3 mutations; they were able to show higher levels of SYK activation in FLT3-ITD-positive AML cells. Though they demonstrated different levels of SYK kinase activity, the exact mechanism for this observation is still unknown. In our study, however, all 5 patients with FLT3 mutations progressed rapidly during the first cycle. Further testing would need to be conducted to determine if the tyrosine kinase inhibition effect by erlotinib is limited to SYK, or if it causes differentiation through multiple kinase inhibition.

In the preclinical data, not all the cell lines responded to erlotinib exposure. Further studies would be required to determine what differences account for inducing differentiation in some cell lines and patient samples and not others. In addition, SYK inhibition may also involve aberrant Lyn [27] or mTOR-mediated signaling [28]. Involvement of other pathways may be synergistic or essential to the primary mechanism. A better understanding of the molecular biology of the anti-AML mechanism of erlotinib is needed for better risk stratification of patients, i.e., those who may respond to treatment with this drug and other tyrosine kinase inhibitors. 
Overall, erlotinib treatment was well tolerated, with most of the adverse events related to the AML, other comorbidities, or concomitant medications. Erlotinib toxicities were generally of mild-to-moderate severity and managed with medications. Since erlotinib was well tolerated, it could be an option for combination therapy if further preclinical studies could elucidate the ideal population in which it would be more likely to exert antileukemic activity.

In conclusion, erlotinib has limited clinical efficacy in patients with refractory or relapsed AML. The population enrolled in this trial was previously very heavily treated, including a high percentage of patients who had received prior HSCT. Still, the outcome is disappointing. Further exploration of erlotinib as a treatment option for AML requires a better understanding of its anti-AML mechanism in the occasional responses, in order to determine the biomarkers that may help predict which patients could potentially benefit from treatment as a single agent or in combination with other agents. If more could be understood about the biology of the anti-AML mechanism, erlotinib and future generations of similar drugs can be revisited as possible therapeutic options.

\section{Disclosure Statement}

J.E.C. has research support from Ariad, Bristol-Myers-Squibb (BMS), Novartis, Pfizer, and Teva, and is a consultant for Ariad, BMS, Pfizer, and Teva. All other authors have no disclosures to declare.

\section{Funding Sources}

This study was supported in part by the MD Anderson Cancer Center and Astellas Pharma Inc.

\section{Author Contributions}

I.A. and P.P. wrote the paper; J.E.C. designed and coordinated the study, treated patients, directed the clinical trial, and edited the manuscript; H.M.K., N.P. E.J., A.F., W.G.W., Z.E., M.K., F.R., Y.A., and G.B. enrolled the patients and conducted the research; and P.P., A.D., and J.R. analyzed the data and performed the statistics. B.L. designed, performed, and analyzed and wrote the results of the experiment corresponding to Figure 3. V.G. participated in writing of the protocol and the manuscript, and in analyzing data in Figure 3. All authors participated in the discussion, and reviewed and approved the current version of the manuscript.

\section{References}

1 Bose P, Vachhani P, Cortes JE: Treatment of relapsed/refractory acute myeloid leukemia. Curr Treat Options Oncol 2017;18:17.

2 Thol F, Schlenk RF, Heuser M, Ganser A: How I treat refractory and early relapsed acute myeloid leukemia. Blood 2015;126: 319-327.

3 Dohner $\mathrm{H}$, Weisdorf DJ, Bloomfield CD: Acute myeloid leukemia. N Engl J Med 2015; 373:1136-1152.

4 Rosell R, Carcereny E, Gervais R, Vergnenegre A, Massuti B, Felip E, et al: Erlotinib versus standard chemotherapy as first-line treatment for European patients with advanced EGFR mutation-positive non-small-cell lung cancer (EURTAC): a multicentre, open-label, randomised phase 3 trial. Lancet Oncol 2012; 13:239-246.

5 Chan G, Pilichowska M: Complete remission in a patient with acute myelogenous leukemia treated with erlotinib for non-small-cell lung cancer. Blood 2007;110:1079-1080.

6 Pitini V, Arrigo C, Altavilla G: Erlotinib in a patient with acute myelogenous leukemia and concomitant non-small-cell lung cancer. J Clin Oncol 2008;26:3645-3646.
7 Stegmaier K, Corsello SM, Ross KN, Wong JS, Deangelo DJ, Golub TR: Gefitinib induces myeloid differentiation of acute myeloid leukemia. Blood 2005;106:2841-2848.

8 Lindhagen E, Eriksson A, Wickstrom M, Danielsson K, Grundmark B, Henriksson R, et al: Significant cytotoxic activity in vitro of the EGFR tyrosine kinase inhibitor gefitinib in acute myeloblastic leukaemia. Eur J Haematol 2008;81:344-353.

9 Boehrer S, Ades L, Braun T, Galluzzi L, Grosjean J, Fabre C, et al: Erlotinib exhibits antineoplastic off-target effects in AML and MDS: a preclinical study. Blood 2008;111:21702180 .

10 Boehrer S, Ades L, Galluzzi L, Tajeddine N, Tailler M, Gardin C, et al: Erlotinib and gefitinib for the treatment of myelodysplastic syndrome and acute myeloid leukemia: a preclinical comparison. Biochem Pharmacol 2008;76:1417-1425.

11 Hahn CK, Berchuck JE, Ross KN, Kakoza RM, Clauser K, Schinzel AC, et al: Proteomic and genetic approaches identify Syk as an AML target. Cancer Cell 2009;16:281-294.

12 Turner M, Schweighoffer E, Colucci F, Di Santo JP, Tybulewicz VL: Tyrosine kinase SYK: essential functions for immunoreceptor signalling. Immunol Today 2000;21:148-154.
13 Gobessi S, Laurenti L, Longo PG, Carsetti L, Berno V, Sica S, Leone G, Efremov DG: Inhibition of constitutive and BCR-induced Syk activation downregulates $\mathrm{Mcl}-1$ and induces apoptosis in chronic lymphocytic leukemia B cells. Leukemia 2009;23:686-697.

14 Quiroga MP, Balakrishnan K, Kurtova AV, Sivina M, Keating MJ, Wierda WG, Gandhi V, Burger JA: B-cell antigen receptor signaling enhances chronic lymphocytic leukemia cell migration and survival: specific targeting with a novel spleen tyrosine kinase inhibitor, R406. Blood 2009;114:1029-1037.

15 Tomasson MH, Xiang Z, Walgren R, Zhao Y, Kasai Y, Miner T, et al: Somatic mutations and germline sequence variants in the expressed tyrosine kinase genes of patients with de novo acute myeloid leukemia. Blood 2008; 111:4797-4808.

16 Cheson BD, Bennett JM, Kopecky KJ, Buchner T, Willman CL, Estey EH, et al: Revised recommendations of the International Working Group for Diagnosis, Standardization of Response Criteria, Treatment Outcomes, and Reporting Standards for Therapeutic Trials in Acute Myeloid Leukemia. J Clin 2003;21: 4642-4649. 
17 Lamothe B, Besse A, Campos AD, Webster WK, Wu H, Darnay BG: Site-specific Lys-63linked tumor necrosis factor receptor-associated factor 6 auto-ubiquitination is a critical determinant of I kappa B kinase activation. J Biol Chem 2007;282:4102-4112.

18 Weber C, Schreiber TB, Daub H: Dual phosphoproteomics and chemical proteomics analysis of erlotinib and gefitinib interference in acute myeloid leukemia cells. J Proteomics 2012;75:1343-1356.

19 Hunter T: A tail of two src's: mutatis mutandis. Cell 1987;49:1-4.

20 Tsang E, Giannetti AM, Shaw D, Dinh M, Tse JK, Gandhi S, et al: Molecular mechanism of the Syk activation switch. J Biol Chem 2008; 283:32650-32659.
21 Thepot S, Boehrer S, Seegers V, Prebet T, Beyne-Rauzy O, Wattel E, et al: A phase I/II trial of erlotinib in higher risk myelodysplastic syndromes and acute myeloid leukemia after azacitidine failure. Leuk Res 2014;38: 1430-1434.

22 Sayar H, Czader M, Amin C, Cangany M, Konig $\mathrm{H}$, Cripe LD: Pilot study of erlotinib in patients with acute myeloid leukemia. Leuk Res 2015;39:170-172.

23 Mahmud H, Kornblau SM, Ter Elst A, Scherpen FJ, Qiu YH, Coombes KR, de Bont ES: Epidermal growth factor receptor is expressed and active in a subset of acute myeloid leukemia. J Hematol Oncol 2016;9:64.

24 Boros K, Puissant A, Back M, Alexe G, Bassil CF, Sinha P, et al: Increased SYK activity is associated with unfavorable outcome among patients with acute myeloid leukemia. Oncotarget 2015;6:25575-25587.
25 Lainey E, Wolfromm A, Marie N, Enot D, Scoazec M, Bouteloup C, et al: Azacytidine and erlotinib exert synergistic effects against acute myeloid leukemia. Oncogene 2013;32: 4331-4342.

26 Puissant A, Fenouille N, Alexe G, Pikman Y, Bassil CF, Mehta S, et al: SYK is a critical regulator of FLT3 in acute myeloid leukemia. Cancer Cell 2014;25:226-242.

27 Komrokji RS, Padron E, Yu D, Fulp WJ, Rodriguez Y, Tinsley S, List AF, Lancet JE: Phase II clinical study of erlotinib for treatment of myelodysplastic syndromes. Am J Hematol 2014;89:809-812.

28 Carnevale J, Ross L, Puissant A, Banerji V, Stone RM, DeAngelo DJ, Ross KN, Stegmaier $\mathrm{K}$ : SYK regulates $\mathrm{mTOR}$ signaling in AML. Leukemia 2013;27:2118-2128. 